\title{
Endovascular Therapy of M2 Occlusion in IMS III: Role of M2 Segment Definition and Location on Clinical and Revascularization Outcomes
}

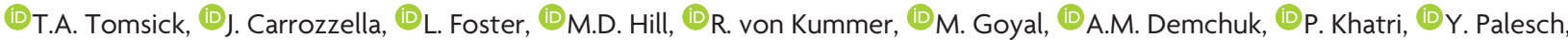 \\ (D).P. Broderick, (1)S.D. Yeatts, and (1D.S. Liebeskind, for the IMS III Investigators
}

\& $\mathrm{O}$ 트

\begin{abstract}
BACKGROUND AND PURPOSE: Uncertainty persists regarding the safety and efficacy of endovascular therapy of M2 occlusions following IV tPA. We reviewed the impact of revascularization on clinical outcomes in 83 patients with M2 occlusions in the Interventional Management of Stroke III trial according to specific M1-M2 segment anatomic features.
\end{abstract}

MATERIALS AND METHODS: Perfusion of any M2 branch distinguished M2-versus-M1 occlusion. Prespecified modified TICl and arterial occlusive lesion revascularization and clinical mRS $0-2$ end points at 90 days for endovascular therapy-treated M2 occlusions were analyzed. Post hoc analyses of the relationship of outcomes to multiple baseline angiographic M2 and M1 subgroup characteristics were performed.

RESULTS: Of 83 participants with M2 occlusion who underwent endovascular therapy, $41.0 \%$ achieved mRS $0-2$ at 90 days, including $46.6 \%$ with modified $\mathrm{TICl} 2-3$ reperfusion compared with $26.1 \%$ with modified $\mathrm{TICl} 0-1$ reperfusion (risk difference, $20.6 \% ; 95 \% \mathrm{Cl},-1.4 \%-42.5 \%$ ). mRS $0-2$ outcome was associated with reperfusion for M2 trunk $(n=9)$ or M2 division $(n=42)$ occlusions, but not for M2 branch occlusions $(n=28)$. Of participants with trunk and division occlusions, $63.2 \%$ with modified $\mathrm{TICl} 2 \mathrm{a}$ and $42.9 \%$ with modified $\mathrm{TICl} 2 \mathrm{~b}$ reperfusion achieved mRS 0 -2 outcomes; mRS 0 -2 outcomes for M2 trunk occlusions (33\%) did not differ from distal (38.2\%) and proximal (26.9\%) M1 occlusions.

CONCLUSIONS: mRS $0-2$ at 90 days was dependent on reperfusion for M2 trunk but not for M2 branch occlusions. For M2 division occlusions, good outcome with modified $\mathrm{TICl} 2 \mathrm{~b}$ reperfusion did not differ from that in modified $\mathrm{TICl} 2 \mathrm{a}$. $\mathrm{M} 2$ segment definition and occlusion location may contribute to differences in revascularization and good outcome between Interventional Management of Stroke III and other endovascular therapy studies.

ABBREVIATIONS: ATA = anterior temporal artery; $\mathrm{EVT}=$ endovascular therapy; IMS = Interventional Management of Stroke; $\mathrm{MID}=\operatorname{distal} \mathrm{MI} ; \mathrm{MIP}=$ proximal MI; $\mathrm{mTICI}=$ modified $\mathrm{TICI}$

$\mathbf{R}$ ecent analysis of M2 occlusions treated by IV tPA and endovascular therapy (EVT) in the Interventional Management of Stroke (IMS) I and II trials and by EVT in the Prolyse in Acute Cerebral Thromboembolism (PROACT) II study failed to dem-

Received June 17, 2016; accepted after revision August 1.

From the Department of Radiology (T.A.T., J.C.), University of Cincinnati Academic Health Center, University Hospital, Cincinnati, Ohio; Department of Biostatistics, Bioinformatics, and Epidemiology (L.F., Y.P., S.D.Y.), Medical University of South Carolina, Charleston, South Carolina; Calgary Stroke Program (M.D.H., A.M.D.), Department of Clinical Neurosciences, Medicine, Community Health Sciences, Hotchkiss Brain Institute, University of Calgary, Foothills Hospital, Calgary, Alberta, Canada; Department of Neuroradiology (R.v.K.), Dresden University Stroke Center, Universitätsklinikum Carl Gustav Carusan deTechnischen Universität Dresden, Dresden, Germany; Department of Radiology and Clinical Neurosciences (M.G.), University of Calgary, Calgary, Alberta, Canada; Department of Neurology (P.K., J.P.B.), University of Cincinnati Academic Health Center, Cincinnati, Ohio; and University of California Los Angeles Stroke Center (D.S.L.), Los Angeles, California.

The work was supported by grants from the National Institutes of Health and the National Institute of Neurological Disorders and Stroke (UC U01NS052220, MUSC Uo1NS054630 and U01NS077304) and by Genentech, EKOS, Concentric Medical, Cordis Neurovascular, and Boehringer Ingelheim. onstrate an association between reperfusion and good outcome. ${ }^{1-4}$ Other recent publications are more optimistic regarding outcome with EVT for M2 occlusion. ${ }^{5-7}$ Uncertainty arises regarding not only patient selection and the utility of EVT for M2 occlusions identified on digital subtraction angiography (DSAM2), ${ }^{8,9}$ but also of the M1-M2 occlusion designation. More recent trials with positive EVT outcomes included very few DSA-M2 occlusions, and some specifically excluded them. ${ }^{10-12}$

We herein summarize the efficacy and safety outcomes of combined IV-EVT in DSA-M2 occlusions in the Interventional Management of Stroke III trial as originally reported, ${ }^{13}$ and we also report post hoc subgroup analyses that explored the hypoth-

Please address correspondence to Thomas A. Tomsick, MD, University of Cincinnati, UC Health, Department of Radiology, 231 Albert Sabin Way, Cincinnati, OH 45267-0762; e-mail: Thomas.Tomsick@UCHealth.com

- Indicates open access to non-subscribers at www.ajnr.org

= Indicates article with supplemental on-line tables.

http://dx.doi.org/10.3174/ajnr.A4979 

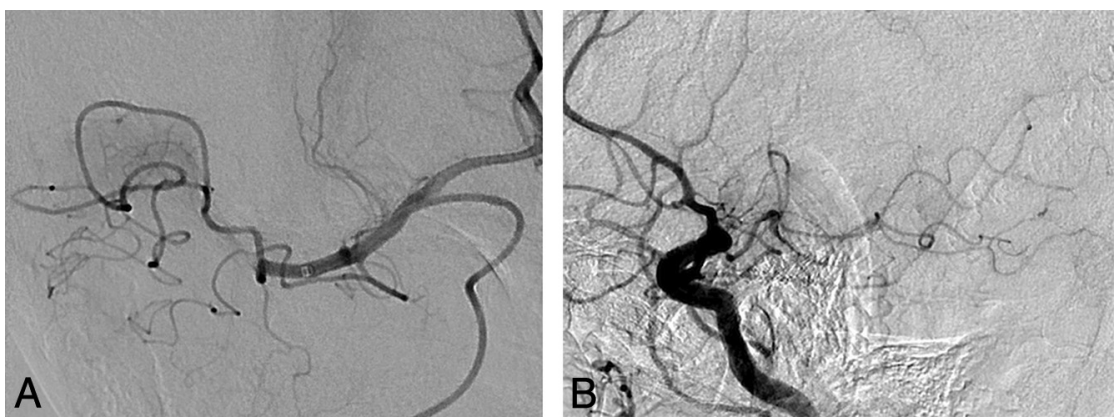

FIG 1. A, Right M1 trunk gives rise to the ATA with the posterior temporal branch filling on microcatheter injection. $B$, Lateral view baseline common carotid arteriogram confirms mid-and posterior temporal lobe cortical supply from the patent posterior temporal artery.
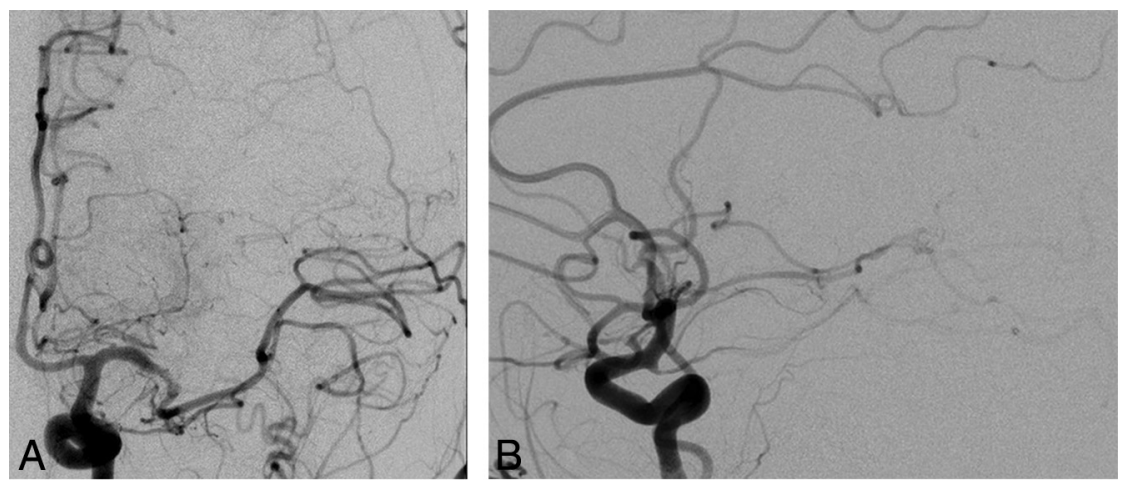

FIG 2. A, Anteroposterior: short M1 trunk with no ATA arising is shown. An isolated M2 holotemporal branch originates, simulating and giving origin to the ATA. It then exits the insular cistern, with multiple middle and posterior temporal arteries draping over and supplying the remainder of the temporal lobe $(B)$. $B$, Lateral view common carotid arteriogram confirms filling of the holotemporal branch, with no other MCA branches filling.

esis that anatomic heterogeneity, operational definitions, and the affected M2 segment location have an effect on outcome in the reperfusion of $\mathrm{M} 2$ occlusion.

\section{MATERIALS AND METHODS}

IMS III eligibility and exclusion criteria, randomization and statistical methods, and results have been previously reported. ${ }^{13-15}$ CT angiography, CT perfusion, and MR angiography and/or perfusion were allowed in centers where they were established as a local standard of evaluation and care.

The IMS III primary outcome measure was a modified Rankin Scale score of 0-2 at 90 days. Secondary efficacy end points were angiographic reperfusion defined as modified Thrombolysis in Cerebral Infarction (mTICI) 2-3 (minimum arteriographic reperfusion $<50 \%$ of the occluded territory at risk) and mTICI $2 \mathrm{~b}-3$ ( $\geq 50 \%$ reperfusion), and recanalization defined as an arterial occlusive lesion recanalization score of 2-3 (partial or complete recanalization with flow). In addition to standard microcatheter thrombolysis, 4 thrombectomy methods were allowed for EVT of M2 occlusions. ${ }^{16-19}$

DSA was reviewed by the angiographic core lab (T.A.T., D.S.L.), and M2 segment and revascularization scores were determined by consensus. mRS $0-2$ outcomes were analyzed according to $\mathrm{mTICI}$ reperfusion results. The relationship of reperfusion to mRS 0-2 and 0-1 outcomes was determined. As in the Emergency Management of Stroke and IMS I and II trials, the opera- tional definition of M1 occlusion was that $100 \%$ of the MCA cortical distribution was at risk, less anterior temporal artery (ATA) supply, with no antegrade M2 branch filling. ${ }^{20-22}$ The corollary of this physiologic definition of M1 occlusion is that filling of $\geq 1$ classic M2 branch then represents M2 occlusion.

Baseline clinical characteristics of 83 evaluable M2 and 135 M1 occlusions proximal (M1P) or distal (M1D) to the lenticulostriate origins were excerpted from case report forms and compared for differences.

Multiple secondary observations of M1 and M2 anatomic features were derived post hoc from baseline and/or posttreatment DSA and recorded, to identify similarities or differences that might discriminate revascularization and clinical outcome.

An isolated branch arising from $\mathrm{M} 1$, adjacent to and with a similar course to the ATA but larger and distributing to the mid- and posterior temporal lobe supply, was termed a "posterior temporal M2 branch" (Fig 1). An isolated branch arising from M1 simulating the ATA but giving origin to the ATA and the mid- and posterior temporal lobes was termed a "holotemporal" M2 branch (Fig 2). The isolated holotemporal or posterior temporal branch might also variously supply portions of the inferior parietal lobe or temporo-occipital region via distal M3 and M4 cortical arteries. The single vessel continuation of $\mathrm{M} 1$ beyond the isolated patent posterior temporal or holotemporal branches is termed the "M2 trunk," which simulates the distal M1 trunk (Fig 3).

A functional IMS M1-M2 anatomic classification, based on pretreatment and posttreatment angiographic findings and relevant clinical correlates, is detailed in On-line Table 1. Occlusion proximal to the lenticulostriate arteries was termed a "proximal M1 occlusion," and occlusion beyond the lenticulostriate arteries, "a distal M1 occlusion." M2 segment occlusions were categorized as trunk, division, division-branch, and/or branch occlusion. M2 trunk occlusion was occlusion of the single large segment beyond the posterior temporal or holotemporal branch (Fig 3). Occlusion of an M2 segment giving rise to $\geq 2$ classic M2 branches was termed "M2 division occlusion." Occlusion of M2 branches arising from divisions was termed "division-branch occlusion." Occlusion of isolated, individual, classic M2 branches arising from the distal M1 (eg, orbitofrontal, operculofrontal, central or Rolondic, angular, parietal, or posterior temporal branches) was termed "M2 branch occlusion."

Post hoc secondary subgroup analyses in 79 evaluable cases, 


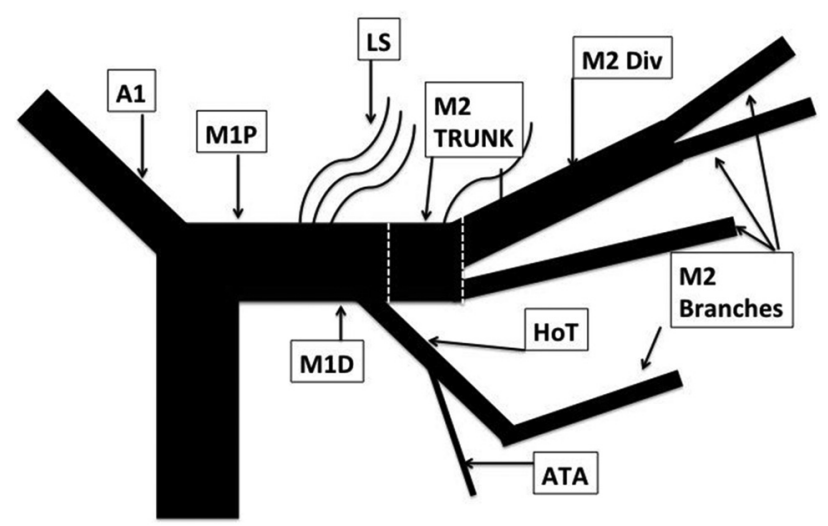

FIG 3. Composite diagram of M1-M2 trunk anatomy based on IMS III post hoc analysis. The M1 trunk proximal to the lenticulostriate arteries (LS) is termed "MIP." The anterior temporal artery arises from the holotemporal M2 branch (HoT). The M2 trunk is a continuation of the distal M1 trunk, beyond a holotemporal (HoT) or posterior temporal M2 branch. The M2 trunk divides into M2 divisions (M2 Div) or branches. M2 divisions divide further into $M 2$ branches.

including review of revascularization and clinical outcome, were also performed, including analysis of the following: 1) occluded segment description (trunk, division, division-branch, branch); 2) estimated percentage MCA distribution of the region at risk according to the occluded segment anatomy; 3) M2 occlusion location (proximal, mid, or distal) and number (single or multiple) on revascularization and outcome; and 4) the presence of isolated holotemporal and posterior temporal lobe branch supply and M2 trunk occlusion on day-2 CTP core infarct and penumbra volumes.

Primary safety end points recorded were mortality and symptomatic intracranial hemorrhage, defined as any intracranial hemorrhage associated with neurologic deterioration within the first 30 hours of IV tPA initiation. Secondary end points included parenchymal hematoma types 1 and 2; asymptomatic intracranial hemorrhage; subarachnoid hemorrhage or intraventricular hemorrhage; angiographically identified vessel dissection or perforation; and DSA-identified emboli into a new (previously unoccluded) arterial territory.

Twenty-six M2 and 45 M1 occlusions from IMS I and II were reviewed post hoc for confirmation of reporting reproducibility and the effect of anatomic characteristics on outcome compared with IMS III.

Differences in reperfusion and mRS 0-2 outcomes between M2 occlusion subgroups were analyzed with the $\chi^{2}$ test, and differences in percentage MCA distribution at risk between subgroups, via the $t$ test.

\section{RESULTS}

Complete patient accountability, clinical, revascularization, and safety results have been previously reported for the entire EVT and IV rtPA groups. ${ }^{13,20}$ No differences in baseline clinical characteristics of 83 subjects with M2 occlusion and 135 previously reported M1 occlusions treated with EVT were identified (Online Table 2). ASPECTS of 8-10 was significantly lower with M1 occlusion proximal to the striate arteries $(P=.02)$. The proportion of subjects with a baseline NIHSS score of $\geq 20$ was not.

Overall, 34/83 (41\%) mRS 0-2 outcomes occurred with M2 EVT. Reperfusion trended $(P=.09)$ toward an association with good outcome (46.7\% mRS $0-2$ for mTICI 2-3 compared with $26.1 \%$ for mTICI $0-1$; risk difference, $20.6 \%$; $95 \%$ CI $-1.4 \%-$ $42.5 \%)$. M2 occlusions were treated predominantly by standard microcatheter thrombolysis ( $n=54,65.1 \%$ ), with 5 sonographyassisted thrombolysis, 13 Merci retriever (Concentric Medical, Mountain View, California), 10 Penumbra System (Penumbra, Alameda, California), and 1 Solitaire Stent (Covidien, Irvine, California) thrombectomy procedures. mTICI 2-3 reperfusion was achieved in 66\% (39/59) of thrombolysis-only treatments and in $88 \%(21 / 24)$ of thrombectomy-device procedures $(P=.048)$.

The Table summarizes M2-segment-occlusion mRS 0-2 outcomes according to mTICI reperfusion results for not only each mTICI grade but also grouped as mTICI $0-1$ versus $2-3$ and as $0-2 \mathrm{a}$ versus $2 \mathrm{~b}-3$. Good outcome for trunk occlusion required mTICI $2 b-3$ reperfusion. No mRS $0-2$ outcome differences were identified for mTCI $2 \mathrm{a}$-versus-2b reperfusion for division occlusion. M2 branch occlusions achieved numerically poorer mTICI 2b-3 reperfusion (28.6\%) and mRS 0-2 outcomes overall $(32.1 \%)$ compared with trunk and division occlusions.

On post hoc review of M2 occlusions, ATAs were identified arising from $42(53.2 \%) \mathrm{M} 1$ and $34(43.0 \%)$ M2 vessels in 79 evaluable subjects, either before or after revascularization. Eighteen $(22.8 \%)$ holotemporal $(n=10)$ and posterior temporal $(n=$ 8 ) branches arising from M1 and simulating the ATA were identified on baseline DSA, half with other patent M2 segments. Seven holotemporal and 2 posterior temporal branches were the only M2 branches patent, defining M2 trunk occlusion and closely simulating M1 trunk occlusion in appearance. Lenticulostriate arteries arose from 16 (20.3\%) M2 segments overall, but in association with $5(50 \%)$ patent holotemporal arteries and $3(33.3 \%)$ M2 trunk occlusions.

On the basis of these anatomic features and the IMS III definitions, the core lab estimated that $30.4 \%$ of M2 occlusions could have been termed M1 occlusion, predominantly where posterior temporal or holotemporal branches simulated the ATA (10/18 versus $14 / 61, P=.008)$. One instance of patency of an orbitooperculofrontal division as the only patent segment, without ATA, was classified as an M2 division occlusion, with no ATA or other M2 segments filling from a large trunklike segment.

Post hoc analysis of revascularization and clinical outcomes for trunk, division, division-branch, and branch occlusions and for proximal and distal M1 occlusion is detailed in On-line Table 3. mRS outcome $0-2$ for M2 occlusion was similar to that for M1D, despite numerically lower mTICI $2-3$ and $2 b-3$ reperfusion. M2-plus-M1D occlusions combined had a higher proportion of mRS $0-1$ and $0-2$ outcomes compared with proximal M1 occlusion ( $P=.06$ and 0.07 , respectively). Good outcome for M2 trunk occlusion $(33.3 \%)$ was numerically greater than for M1P occlusion (26.9\%, $P>.05)$, but not for M1D (38.2\%, $P>.05)$.

M2 trunk occlusions were associated with a greater percentage area distribution at risk for infarction compared with division and branch occlusions combined $(P=.0012)$, as were division occlusions compared with branch occlusions $(P<.0001)$. No differ- 
mRS 0-2 outcomes for 79 evaluable M2 trunk, division, and branch occlusion grouped according to $\mathrm{mTICI} 0-1,2 \mathrm{a}, 2 \mathrm{~b}-3$ reperfusion grade $^{\mathrm{a}}$

\begin{tabular}{|c|c|c|c|c|c|c|}
\hline \multirow[b]{2}{*}{ mTICI Grade } & \multicolumn{2}{|c|}{ M2 Trunk $(n=9)$} & \multicolumn{2}{|c|}{ M2 Division $(n=42)$} & \multicolumn{2}{|c|}{ M2 Branch $(n=28)$} \\
\hline & $N$ & $\%$ mRS 0-2 & $N$ & $\% \mathrm{mRS} 0-2$ & $N$ & $\% \mathrm{mRS} 0-2$ \\
\hline $0-1$ & 2 & 0 & 6 & 0 & 11 & 36.4 \\
\hline $2 a$ & 1 & 0 & 18 & 66.7 & 9 & 22.2 \\
\hline $2 b-3$ & $6^{b}$ & 50 & $18^{b}$ & 44.4 & 8 & 37.5 \\
\hline
\end{tabular}

a This table uses only the evaluable sample.

${ }^{\mathrm{b}} \mathrm{mTICI}$ 3: $n=1$.

ence in MCA distribution at risk was estimated between 16 division-branch and 12 branch occlusions, but excellent and good outcomes were numerically fewer for division-branch occlusions.

Fifty-four of 79 (69.1\%) DSA-M2 occlusions were proximal, with greater estimated percentage MCA distribution at risk compared with mid- or distal occlusions $(P=.0001)$. Ten $(12.7 \%)$ multiple M2 occlusions included 6 divisions with additional branch occlusions and 4 multiple branch occlusions. The estimated percentage MCA at risk was greater for multiple M2 occlusions than for single ones $(P=.05)$.

Post hoc review of anatomic features of 27 M2 and 45 M1 previously treated IMS I and II occlusions confirmed classification consistency with IMS III, including patent holotemporal branches in $3 \mathrm{M} 2$ trunk occlusions (11.1\%). Fifty percent of division-branch or branch occlusions were confirmed in IMS I and II, compared with $37.1 \%$, in IMS III. In the 3 IMS studies, 5/12 (41.6\%) M2 trunk occlusions achieved mRS 0-2 outcomes.

Symptomatic intracranial hemorrhage occurred in 7.2\% (6/83) of subjects with IMS III M2 occlusions. One (1.2\%) arterial perforation and 3 (3.6\%) emboli into a new (previously unoccluded) arterial territory were identified by the core lab. Ten deaths $(12.0 \%)$ occurred.

\section{DISCUSSION}

In IMS III, as in IMS I and II, among DSA-defined M2 occlusions, independent functional outcome (mRS 0-2) was not associated with mTICI $2 \mathrm{~b}-3$ reperfusion compared with EVT of the more proximal intracranial ICA or M1 occlusions. ${ }^{20}$ No good outcomes for trunk occlusion occurred in the absence of mTICI $2 b-3$ reperfusion. No differences in good outcome were identified for mTICI 2a-versus-2b reperfusion for division occlusion. Good outcome was not associated with reperfusion in branch occlusions; this finding may be due to the small sample size $(n=29)$, limited amount of at-risk tissue, or recanalization spontaneously or by IV rtPA alone after completion of EVT. While IMS III M2 mTICI 2-3 reperfusion was better than that in IMS I and II (72.3\% versus $60.9 \%)$, mRS $0-2$ outcomes were poorer $(40.9 \%$ versus $69.6 \%) .{ }^{1,4,9}$ Good outcomes for M2 occlusion with mTICI $0-1$ reperfusion were more frequent in IMS I and II (26.1\% versus $77.7 \%$ ), all occurring with branch occlusions.

In addition to differences in M2 segment occlusion types treated, differences in the definition of M1 and M2 occlusion may contribute to reported outcome differences. ${ }^{23,24}$ A similar ratio of M2-to-M1 occlusion in IMS I and II (64.4\%) and IMS III (60.7\%) suggests comparable general definition application. Whereas PROACT II had a similar percentage ratio $(62.2 \%)$, the ratio was lower $(40.7 \%)$ in the Middle Cerebral Artery Embolism Local Fibrinolytic Intervention Trial (MELT). ${ }^{25}$ The definition of M1 versus M2 has historically been based on anatomic criteria, with the hori- zontal sphenoidal MCA segment in the stem of the Sylvian fissure, proximal to the insula, termed M1, irrespective of the atrisk proportion of the brain supplied by the occluded segment. ${ }^{26}$ The IMS operational functional definition of M1 occlusion places virtually its entire cortical distribution at risk and allows confident comparability of baseline occlusion and outcome data among the IMS studies.

The classic clinicoanatomic model of 2 M2 divisions (superior/anterior or inferior/posterior) occurs in approximately $50 \%$ of instances of M1 trunk branching, pseudo-bifurcations, and -trifurcations, each in approximately $25 \%$ of patients. ${ }^{27,28}$ The description of postdivision branching has not been uniform historically. Where M2 "division" occlusion therapy in PROACT II included treatment of not only divisions that will branch but also branches from divisions and individual branches, potential confusion in terminology exists. Standard arteriographic references avoid "division" entirely, refer to groups or complexes, mix "branch" and "artery" somewhat interchangeably, and have used the term "trunk" to vaguely describe a large Sylvian segment beyond an operculofrontal branch terminating in parietal and angular branches. ${ }^{29}$ Muddled terminology risks including, lumping, equating, and then comparing occluded classic M2 division occlusions $(53.1 \%)$ with smaller, single, even distal M2 divisionbranch $(19.8 \%)$ or branch occlusions $(16.0 \%)$ or major trunks beyond a single patent, classic M2 branch (11.1\%) in stroke-treatment studies.

Two nomenclature adaptions are used here for post hoc description and analysis of observations initially made on primary core lab reporting. The holotemporal branch supplying the entire temporal lobe was linked to M2 "trunk" occlusion when no other M2 branches were filling, simulating M1 occlusion. Designation of any M2 branch patency determining the occluded segment is also termed "M2" as either trunk, single or multiple divisions, division-branches, or branches.

If IMS M2 trunk occlusion is attributed to the M1 rather than M2 group, 9.0\% good outcome difference between IMS M2 trunk and IMS III M1 occlusion (5/12, 41.6\% versus 44/135, 32.6\%) would increase reported good outcomes for M1 compared with M2 occlusion. Ascribing an M1 occlusion instead of an M2 trunk occlusion may falsely reduce anticipated brain volume at risk, erroneously increase the expected risk of temporal lobe edema and herniation, and underestimate available collateral flow while overestimating collateral need, thereby predisposing to a higher percentage of good outcome compared with M1 occlusion as defined in IMS. A single branch supplying the entire temporal lobe has been suggested by Gibo et $\mathrm{al}^{27}$ to occur anatomically in $2 \%$ but was identified in $10(13.6 \%)$ in our DSA cohort. Alexandrov has identified a prominent ATA acting as a collateral with transcranial Doppler (A. Alexandrov, MD, personal oral communication, International Stroke Conference, February 12, 2014). Menon et al ${ }^{30}$ identified a patent "prominent anterior temporal artery" on 20/ $102(19.6 \%)$ CTAs in patients with M1 occlusion. Survival was better in the presence of its demonstration $(18 / 20,90 \%)$ than in its absence $(66 / 82,80.4 \%)$. While the relationship of their demonstration of a prominent ATA to our holotemporal/posterior 
temporal branch designation is uncertain, it is reasonable to hypothesize that the assignment of the latter as a marker of the functional M1-M2 junction has some relevance as EVT refines its methods and metrics beyond revascularization alone in exploring differences in outcomes in EVT. Post hoc blinded review of limited CT perfusion studies in IMS III found that mean core and penumbra volumes were numerically lowest in M2 trunk versus M2 division versus M1 trunk occlusions: 4.0 versus 17.2 versus $18.6 \mathrm{~mL}$ for core, and 27.8 versus 62.3 versus $85.7 \mathrm{~mL}$ for penumbra, respectively. ${ }^{31}$

The percentage MCA at risk was greater with M2 trunk occlusion than in its absence, as was division occlusion with divisionbranch and branch occlusion. However, the percentage MCA at risk, mTICI 2-3, and mRS 0-1 and 0-2 were lower with branch than division trunk occlusion. This contradiction requires further analysis of baseline CT and CTA imaging findings to determine whether more proximal occlusions may have already futilely recanalized, leaving only residual branch occlusion before DSA.

Limitations exist in these observations and analyses. Data on M2 occlusion here are based on EVT following IV rtPA administration. Results were obtained with thrombectomy technology and thrombolytic methods not commonly used currently. Whereas up to $20 \%$ of initially occluded arteries may have recanalized before angiography, clinical outcomes may relate more to the original occlusion than to the arteriographic occlusion. ${ }^{20,32}$ In subjects with M2 occlusion on baseline CTA, no significant difference in patency was identified on 24 -hour CTA $(88.5 \%$ in the EVT arm versus $76.5 \%$ in the IV tPA arm), but 90 -day mRS $0-2$ outcomes were greater with EVT $(n=31)$ than IV tPA alone $(n=$ $15)$ in M2 subjects with no ICA occlusion/stenosis, $51.6 \%$ versus $33.4 \% .^{33}$

Reperfusion results were preliminarily reported here variously as mTICI 2-3 and/or 2b-3 to allow comparison with IMS I and II results of failed reperfusion versus outcome. Neither interobserver agreement in distinguishing $2 \mathrm{a}$ versus $2 \mathrm{~b}$ reperfusion nor mTICI $2 b-3$ reperfusion as a predictor of good outcome for M2 occlusion had yet been shown. The latter is not confirmed here for division or branch occlusion, and no difference in mRS $0-2$ outcome between mTICI $2 \mathrm{a}$ versus $2 \mathrm{~b}$ for division occlusion was identified with reperfusion methods used (Table).

The major limitation of the data and explorative analysis presented here may be perceived in its derivation within the futile IMS IV-EVT treatment paradigm and the treatment methods used. Although thrombectomy devices may be more effective than thrombolysis alone across the M2 group, larger studies with newer devices are indicated for M2 occlusion. Emphasizing terms such as "M2 trunk" and "holotemporal artery" would be irrelevant had not retrospective analyses demonstrated that the unique anatomic features might confer potential relevant differences in brain at risk and/or outcome. Comparisons of small subgroup numbers here, such as the M2 trunk group, are subject to error. Uncommon occlusions beyond the patency of other single segments (such as orbitofrontal or operculofrontal branches) may also be classified as trunk occlusions in the future.

However, the operational IMS occlusion model, excluding M1 occlusion if any M2 branch is filling, reduces the subjectivity of vessel orientation/course, eliminates dilemmas in identifying and classifying major branch points, and becomes one approach to assuring uniformity in outcome analysis for not only M1 occlusion primarily but also M2 segment occlusion secondarily.

\section{CONCLUSIONS}

In IMS III, revascularization rates were higher but mRS $0-2$ outcomes were lower for combined IV rtPA-EVT for M2 occlusion than those measured in IMS I and II. mRS 0-2 outcomes differed according to the involved segment, dependent on mTICI $2 \mathrm{~b}$ reperfusion for trunk occlusion, with no difference between mTICI $2 \mathrm{a}$ and $2 \mathrm{~b}$ reperfusion for division occlusion. mRS $0-2$ outcome was not dependent on reperfusion for M2 branch occlusion. Differences in good outcome between the M2 trunk in IMS I, II, and III (41.6\%) versus M1 occlusion (32.6\%) suggest that failing to distinguish between them could influence reported outcome differences in EVT studies. M2 trunk occlusion, simulating M1 trunk occlusion, is proposed as an M2 occlusion subgroup for closer analysis in EVT studies.

Disclosures: Thomas A. Tomsick—RELATED: Grant: National Institutes of Health*; Support for Travel to Meetings for the Study or Other Purposes: IMS III; UNRELATED: Expert Testimony: medicolegal consulations (no travel). Janice Carrozzella—RELATED: Grant: National Institutes of Health-National Institute of Neurological Disorders and Stroke, Comments: IMS III trial*; Support for Travel to Meetings for the Study or Other Purposes: IMS III; UNRELATED: Employment: Department of Radiology, University of Cincinnati. Lydia Foster-RELATED: Grant: National Institute of Neurological Disorders and Stroke.* Michael D. Hill—RELATED: Grant: National Institute of Neurological Disorders and Stroke grant for the IMS III trial*; UNRELATED: Consultancy: for Merck for an advisory panel for clinical trials; Grants/Grants Pending: Medtronic, Bayer Canada, Boehringer Ingelheim, Comments: grants for clinical trials*; Payment for Lectures including Service on Speakers Bureaus: Boehringer Ingelheim, Bayer Canada, Bristol-Myers Squibb-Pfizer, Comments: honoraria for Continuing Medical Education lectures; Patents (Planned, Pending, or Issued): patent for stroke imaging, Comments: patent pending; Stock/Stock Options: Calgary Scientific Inc, Comments: stock ownership in imaging software company. Mayank GoyalRELATED: Grant: Medtronic, Comments: part funding for the ESCAPE trial, funding for HERMES collaboration*; Consulting Fee or Honorarium: Medtronic, Stryker, MicroVention, Comments: education and advice related to acute stroke treatment and products; UNRELATED: Patents (Planned, Pending, or Issued): GE Healthcare, Comments: licensing agreement for Systems of Stroke Diagnosis. R. von KummerRELATED: Support for Travel to Meetings for the Study or Other Purposes: National Institutes of Health; UNRELATED: Consultancy: Lundbeck A/S, Covidien, Synarc Inc, BrainsGate, Boehringer Ingelheim, Comments: Steering Committee for Desmoteplase in Acute Ischemic Stroke 3/4, Data and Safety Monitoring Board for SWIFT PRIME, Adjudication Committee for Desmoteplase in Acute Ischemic Stroke 3/4, Data and Safety Monitoring Board for IMPact, Data and Safety Monitoring Board for ReSPECT ESUS. Andrew M. Demchuk-RELATED: Grant: National Institutes of Health-National Institute of Neurological Disorders and Stroke, Comments: The IMS III trial was funded by National Institutes of Health-National Institute of Neurological Disorders and Stroke. The CT imaging core lab was supported by funds from the National Institutes of Health-National Institute of Neurological Disorders and Stroke*; UNRELATED: Payment for Lectures including Service on Speakers Bureaus: Medtronic, Comments: honoraria for Continuing Medical Education events. Pooja Khatri-RELATED: Grant: National Institutes of Health-National Institute of Neurological Disorders and Stroke U01 for IMS III*; UNRELATED: Consultancy: Grand Rounds Experts, Comments: On-line clinical consultation; Expert Testimony: medicolegal consultations; Royalties: UpToDate, Comments: On-line publication; Other: Genentech, Penumbra, and Biogen, Comments: Genentech pays my salary for my effort as Lead Principal Investigator of the PRISMS trial. Penumbra has paid my salary for my effort as the Lead Neurology Principal Investigator of the THERAPY trial. Biogen has paid my institution and now pays me for effort as a Data and Safety Monitoring Board member.* Yuko Palesch—RELATED: Grant: National Institutes of Health-National Institute of Neurological Disorders and Stroke, Comments: U01 grant support for IMS III*; UNRELATED: Travel/Accommodations/Meeting Expenses Unrelated to Activities Listed: 13th International Symposium on Thrombolysis Thrombectomy and Acute Stroke Therapy, Comments: travel/accommodation expenses to be a speaker; Other: BrainsGate, Comments: statistical member of the Data and Safety Monitoring Board for their clinical trials. Joseph P. BroderickRELATED: Support for Travel to Meetings for the Study or Other Purposes: Boehringer Ingelheim*; Fees for Participation in Review Activities such as Data Monitoring Boards, Statistical Analysis, Endpoint Committees, and the Like: IMS III; Other: Ge- 
nentech, study medication; EKOS, Concentric Inc, and Cordis Neurovascular supplied study catheters in early phase of the IMS III trial; UNRELATED: Other: Genentech, financial support paid to the department for role on Steering Committee for PRISMS Trial. Sharon D. Yeatts—RELATED: Grant: National Institute of Neurological Disorders and Stroke, Comments: IMS III*; UNRELATED: Consultancy: Genentech, Comments: I received consultant fees for my role on the PRISMS Trial Steering Committee. David S. Liebeskind_UNRELATED: Consultancy: Stryker, Medtronic, Comments: imaging core lab. *Money paid to the institution.

\section{REFERENCES}

1. Rahme R, Yeatts SD, Abruzzo T, et al. Early reperfusion and clinical outcome in patients with M2 occlusion: pooled analysis of the PROACT II, IMS, and IMS II studies. J Neurosurg 2014;121: 1354-58 CrossRef Medline

2. IMS Study Investigators. Combined intravenous and intra-arterial recanalization for acute ischemic stroke: the Interventional Management of Stroke Study. Stroke 2004;35:904-11 CrossRef Medline

3. The IMS II Trial Investigators. The Interventional Management of Stroke (IMS) II study. Stroke 2007;38:2127-35 CrossRef Medline

4. Tomsick TA, Broderick J, Carrozella J, et al; Interventional Management of Stroke II Investigators. Revascularization results in the Interventional Management of Stroke II Trial. AJNR Am J Neuroradiol 2008;29:582-87 CrossRef Medline

5. Galimanis A, Jung S, Mono ML, et al. Endovascular therapy of 623 patients with anterior circulation stroke. Radiology 2012;43: 1052-57 CrossRef Medline

6. Sheth S, Saver J, Jahan R, et al; UCLA Comprehensive Stroke Center. M2 occlusions as targets for endovascular therapy: comprehensive analysis of diffusion/perfusion MRI, angiography, and clinical outcomes. J Neurointerv Surg 2015;7:478-83 CrossRef Medline

7. Flores A, Tomasello A, Cardona P, et al; Catalan Stroke Code and Reperfusion Consortium Cat-SCR. Endovascular treatment for M2 occlusions in the era of stentrievers: a descriptive multicenter experience. J Neurointerv Surg 2015;7:234-37 CrossRef Medline

8. Tomsick TA, Khatri P, Jovin T, et al; IMS III Executive Committee. Equipoise among recanalization strategies. Neurology 2010;74: 1069-76 CrossRef Medline

9. Rahme R, Abruzzo T, Martin RH, et al. Is intra-arterial thrombolysis beneficial for M2 Occlusions? Subgroup analysis of the PROACT II Trial. Stroke 2013;44:240-42 CrossRef Medline

10. Goyal M, Demchuk AM, Menon BK, et al; ESCAPE Trial Investigators. Randomized assessment of rapid endovascular treatment of acute stroke. $N$ Engl J Med 2015;372:1019-30 CrossRef Medline

11. Campbell BC, Mitchell PJ, Kleinig TJ, et al; EXTEND-IA Investigators. Endovascular therapy for ischemic stroke with perfusion-imaging selection. $N$ Engl J Med 2015;372:1009-18 CrossRef Medline

12. Berkheimer OA, Fransen PS, Beumer D, et al; MR CLEAN Investigators. A randomized trial of intraarterial treatment for acute stroke. N Engl J Med 2015;372:11-20 CrossRef Medline

13. Broderick JP, Palesch YY, Demchuk AM, et al; Interventional Management of Stroke (IMS) III Investigators. Endovascular therapy after intravenous rt-PA alone for stroke. N Engl J Med 2013;368:893903 CrossRef Medline

14. Khatri P, Hill M, Palesch Y, et al; Interventional Management of Stroke III Investigators. Methodology of the Interventional Management of Stroke III trial. Int J Stroke 2008;3:130-37 CrossRef Medline

15. Yeatts SD, Martin RH, Foster LD, et al. Challenges of decision-making regarding futility in a randomized trial: the IMS III experience. In: Proceedings of the International Stroke Conference, Honolulu. Hawaii. February 6-8, 2013

16. Smith WS, Sung G, Starkman S, et al; MERCI Trial Investigators. Safety and efficacy of mechanical embolectomy in acute ischemic stroke: results of the MERCI trial. Stroke 2005;36:1432-38 CrossRef Medline

17. Smith WS, Sung G, Saver J, et al. Mechanical thrombectomy for acute ischemic stroke: final results of the Multi MERCI trial. Stroke 2008;39:1205-12 CrossRef Medline

18. Penumbra Pivotal Stroke Trial Investigators. The Penumbra Pivotal Stroke Trial: safety and effectiveness of a new generation of mechanical devices for clot removal in intracranial large vessel occlusive disease. Stroke 2009;40:2761-68 CrossRef Medline

19. Dávalos A, Pereira VM, Chapot R, et al. Retrospective multicenter study of Solitaire FR for revascularization in the treatment of acute ischemic stroke. Stroke 2012;43:2699-705 CrossRef Medline

20. Tomsick T, Yeatts SD, Liebeskind D, et al; IMS III Investigators. Endovascular revascularization results in IMS III: intracranial ICA and M1 occlusions. J Neurointervent Surg 2015;7:795-802 CrossRef Medline

21. Morris PP, Choi IS. Cerebral vascular anatomy. Neuroimaging Clin N Am 1996;6:541-60 Medline

22. Ring BA. The middle cerebral artery. In: Newton TH, Potts DG, eds. Radiology of the Skull and Brain: Angiography. Vol 2. Great Neck: CV Mosby; 1974:1442-78

23. Appireddy RM, Menon BK, Horn M, et al. Using the M2 vessel diameter and baseline NIHSS to identify which M2 occlusions should be treated endovascularly. Stroke 2015;46:AWP56

24. Gulati D, Ducruet A, Aghaebrahim A, et al. Impact of differences in definition of M1 and M2 segment of middle cerebral artery on acute stroke endovascular therapy. Stroke 2015; 46:AWMP22

25. Ogawa A, Mori E, Minematsu K, et al. Randomized trial of intraarterial infusion of urokinase within 6 hours of middle cerebral artery stroke: the middle cerebral artery embolism local fibrinolytic intervention trial (MELT) Japan. Stroke 2007;38:2633-39 CrossRef Medline

26. Zaidat OO, Yoo AJ, Khatri P, et al; Cerebral Angiographic Revascularization Grading (CARG) Collaborators, STIR Revascularization Working Group, STIR Thrombolysis in Cerebral Infarction (TICI) Task Force. Recommendations on angiographic revascularization standards for acute ischemic stroke: a consensus statement. Stroke 2013;44:2650-63 CrossRef Medline

27. Gibo H, Carver CC, Rhoton AL Jr, et al. Microsurgical anatomy of the middle cerebral artery. J Neurosurg 1981;54:151-69 CrossRef Medline

28. Krayenbuhl HA, Yasargil MG. Cerebral Angiography. 2nd ed. Philadelphia; Lippincott; 1968

29. Ring BA. The middle cerebral artery. In: Newton TH, Potts DG, eds. Radiology of the Skull and Brain: Angiography. Vol 2. Great Neck; CV Mosby; 1974:1459

30. Menon BK, Bal S, Modi J, et al. Anterior temporal artery sign in CT angiography predicts fatal brain edema and mortality in acute M1 middle cerebral artery occlusion. J Neuroimaging 2012;22:145-48 CrossRef Medline

31. Livorine A, Vagal A, Shu J, et al. Anatomical variation of M2 occlusions and tissue at risk in IMS III trial: an exploratory analysis. In: Proceedings of the Annual Meeting of American Roentgen Ray Society, Toronto, Ontario, Canada. April 19-24, 2015

32. Von Kummer R, Demchuk AM, Foster LD, et al. Early arterial recanalization after intra-venous tissue-plasminogen-activator treatment in the Interventional Management of Stroke-3 study. Stroke 2014;45:A74

33. Demchuk AM, Goyal M, Yeatts SD, et al; IMS III Investigators. Recanalization and clinical outcome of occlusion sites at baseline CT angiography in the Interventional Management of Stroke III trial. Radiology 2014;273:202-10 CrossRef Medline 